\title{
Cohesive stresses and size effect in quasi-brittle materials
}

\author{
V E SAOUMA $^{1}$ and D NATEKAR ${ }^{2}$ \\ ${ }^{1}$ Department of Civil, Environmental and Architectural Engineering, and \\ ${ }^{2}$ Department of Mechanical Engineering, University of Colorado, Boulder, CO \\ 80309-0427, USA \\ e-mail: saouma@bechtel.Colorado.EDU
}

\begin{abstract}
A novel approach to the derivation of Bažant's size effect law is presented. Contrarily to the original Lagrangian derivation which hinged on energetic consideration, a Newtonian approach based on local stress intensity factors is presented. Through this approach, it is shown that Bažant's size effect law is the first (and dominant) term in a series expansion for the nominal stress. Furthermore, analytical expressions for $B$ are derived for selected specimen geometries.
\end{abstract}

Keywords. Size effect; fracture mechanics; cohesive stresses; cementitious material.

\section{Introduction}

Crack bridging occurs when cohesive stresses join the opposite faces of a crack, shielding the tip from the full effect of the applied load and thus giving rise to increased fracture resistance. Cohesive stresses are present not only in cementitious materials, but also in ceramics (Saouma et al 2002) and are caused by reinforcing fibres, particles (such as aggregates or inclusions), or simply regions of microscopically irregular crack surfaces causing topological interference.

In the absence of these cohesive stresses, and for linear elastic continuum, we would have a brittle material, otherwise we have a quasi-brittle response. The brittle response can be fully addressed by linear elastic fracture mechanics (LEFM) theory, while quasi-brittle ones require a nonlinear solution.

Cohesive stress models were first suggested by Dugdale (1960) using a constant stress, then Barenblatt (1962) extended it to triangular stresses, and most recently Hillerborg et al (1976) assumed a nonlinear model where the stress is a function of the crack opening. The first two models are most appropriate for metals to account for the presence of a plastic zone, whereas Hillerborg's model is more suited for cementitious material with a fracture process zone.

\subsection{Original derivation}

Bažant \& Planas (1998) presented a unified model for the nominal strength of quasi-brittle material. This simple and elegant equation is asymptotic to plasticity and linear elastic fracture mechanics, while capturing the intermediary quasi-brittle response. 


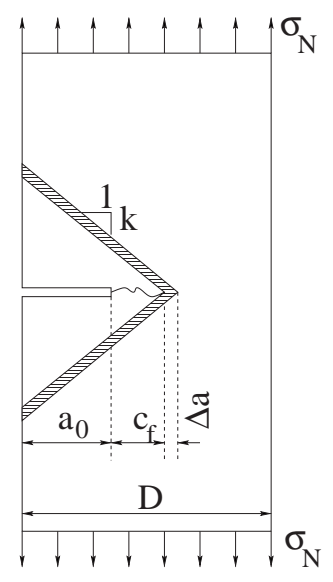

Figure 1. Energy transfer during infinitesimal crack extension.

Considering the energy exchanged during an infinitesimal crack extension in a plate of width $b$ (figure 1), the energy released can be approximated by $b 2 k\left(a_{0}+c_{f}\right) \Delta a \sigma_{N}^{2} / 2 E$, which must be equal to the energy consumed during crack growth: $b G_{F} \Delta a$, hence

$$
b 2 k\left(a_{0}+c_{f}\right) \Delta a \sigma_{N}^{2} / 2 E=b G_{F} \Delta a
$$

yielding

$$
\sigma_{N}=B f_{t}^{\prime} /\left[1+\left(D / D_{0}\right)\right]^{1 / 2}
$$

where,

$$
B f_{t}^{\prime}=\left[G_{f} E / k c_{f}\right]^{1 / 2} \text { and } D / D_{0}=a_{0} / c_{f}=\beta .
$$

In his original paper, Bažant (1984) noted that the analytical or numerical derivation of $B$ and $\beta$ is too difficult, and they are best obtained through statistical regression analysis of test data.

This derivation would be characterized as semi-analytical, since it combines an analytical derivation (albeit with some simplifying assumptions), and experimental derivation of constants too complicated to be derived analytically. Finally, this derivation does not explicitly reference a plasticity and/or a linear elastic fracture mechanics solution. Yet, those two solutions are ultimately asymptotic to the derived size effect law.

\section{Analytical derivation}

In this section, an alternate derivation of the size effect law is presented. Whereas it hinges (for simplifying reasons) on Dugdale's or Barenblatt's model for the cohesive stresses (in lieu of Hillerborg's fracture energy), it will be shown that a purely analytical expression could be derived, hence problem specific numerical values of $B$ can be obtained.

This approach is based on classical elasto-plastic fracture mechanics, (Broek 1986), where in its simplest form the stress intensity factors caused by the cohesive stresses (in a plastic zone or process zone), are assumed to cancel the ones caused by the far field load. Hence, contrary to the original derivation by Bažant (1984), the size effect law will be shown to have explicit roots in plasticity and linear elastic fracture mechanics theories. 


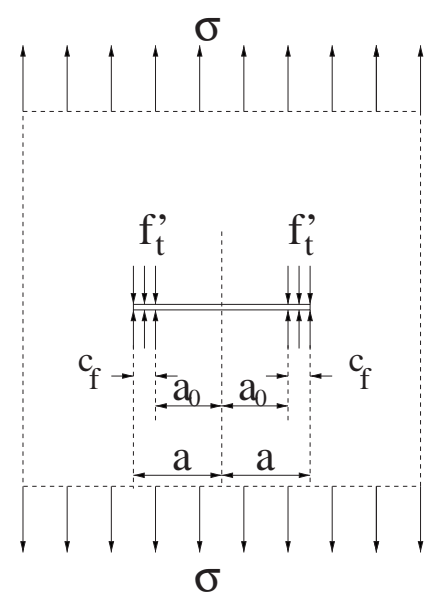

Figure 2. Central crack with Barenblatt's model.

\subsection{Central crack, Dugdale}

Starting with a general case, we consider an infinite plate subjected to a far field uniform tensile stress $\sigma$ and a crack of length $2 a$, at the tip of which we have a uniform cohesive compressive stress equal to the tensile strength $f_{t}^{\prime}$ (figure 2 ).

The stress intensity factors due to the far field and cohesive stresses are:

$$
\begin{aligned}
& K_{a}=\sigma(\pi a)^{1 / 2}, \\
& K_{b}=f_{t}^{\prime}(\pi a)^{1 / 2}\left(1-(2 / \pi) \arcsin \left[\left(a-c_{f}\right) / a\right]\right),
\end{aligned}
$$

respectively (Cherepanov 1979).

Equating those two stress intensity factors, we obtain the nominal strength

$$
\sigma_{n}=f_{t}^{\prime}\left[1-(2 / \pi) \arcsin \left(1-\left(c_{f} / a\right)\right)\right] .
$$

In the limit, for small sizes when $a \simeq c_{f}, \sigma_{n}$ approaches asymptotically $f_{t}^{\prime}$. On the other hand, for large sizes, $c_{f} \simeq 0, \sigma_{n}$ will asymptotically approach zero.

Whereas the expression of $\sigma_{n}$ appears to have the same limits as the size effect law, it is not mathematically similar to it. This will only become apparent if one takes a series expansion of the arcsin function, and substitute $c_{f} / a$ by $s$ :

$$
\begin{aligned}
\sigma_{n} & =\sigma_{n}=f_{t}^{\prime}[1-(2 / \pi) \arcsin (1-s)], \\
& \simeq \frac{2 \sqrt{2} f_{t}^{\prime}}{\pi} s^{1 / 2}+\frac{f_{t}^{\prime}}{3 \sqrt{2} \pi} s^{3 / 2}+\frac{3 f_{t}^{\prime}}{40 \sqrt{2} \pi} s^{5 / 2}+O[s]^{7 / 2} .
\end{aligned}
$$

Neglecting the terms of power greater than 1 (since $s$ is at most equal to 1 ), and substituting $s=1 /(1+r)$ where $r=a_{0} / c_{f}$, we obtain

$$
\sigma_{n}=(2 \sqrt{2} / \underbrace{\pi}_{B}) f_{t}^{\prime}[1 /(1+\underbrace{r}_{\beta})]^{1 / 2} .
$$

We have thus recovered the size effect law as originally derived by Bažant as expressed by (2) with the additional benefit that $B$ is quantified for this combination of geometry and cohesive stresses (figure 3). 

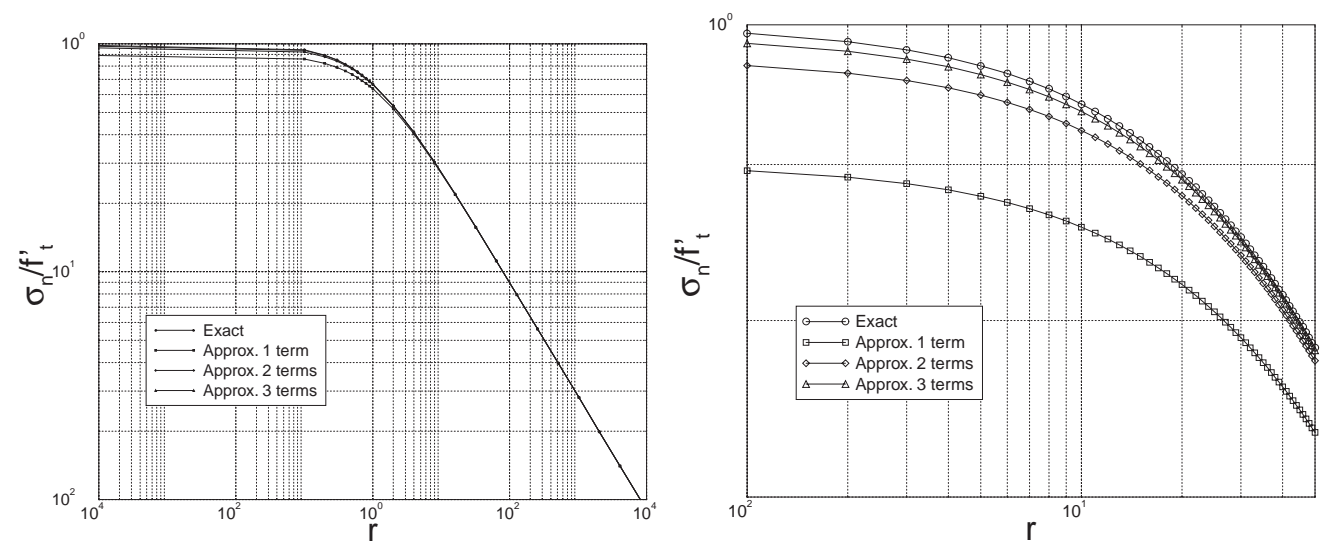

Figure 3. Nominal strength in terms of size for a centre crack plate with Dugdale's cohesive stresses.

\subsection{Edge crack, Dugdale}

We next consider an arbitrary geometry of a cracked structure whose stress intensity factor due to the far field stress can be expressed as

$$
K_{a}=\alpha \sigma(\pi a)^{1 / 2},
$$

where $\alpha$ is a coefficient which accounts for geometry, boundary conditions, and load.

Alternatively, the stress intensity factor corresponding to a constant stress, figure 4 is given by Stevens \& Guiu (1994) as

$$
K_{b}=-f_{t}^{\prime}(\pi a)^{1 / 2} f_{b}
$$

and

$$
\begin{aligned}
f_{b}= & 0.903 s^{1 / 2}+1 / 3\left(0.4406 s^{3 / 2}\right)+1 / 5\left(0.4997 s^{5 / 2}\right)-1 / 7\left(0.1438 s^{7 / 2}\right) \\
& -1 / 9\left(0.04578 s^{9 / 2}\right),
\end{aligned}
$$

where $s=c_{f} / a$. Since at most $s=1$, we keep only the first term which has an exponent lower than one.

Equating $K_{a}$ to $K_{b}$, we solve for the far field nominal stress $\sigma_{N}$ which would result in a zero net stress intensity factor at the tip of the crack. For this problem, we obtain

$$
\sigma_{N}=(0.903 / \alpha)\left[f_{t}^{\prime}(s)^{1 / 2}\right] \text {. }
$$

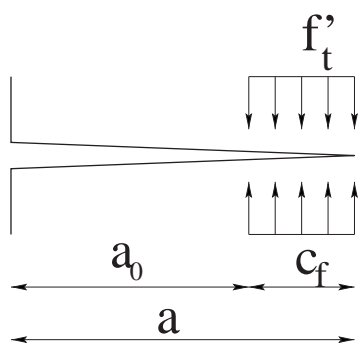

Figure 4. Dugdale's model. 


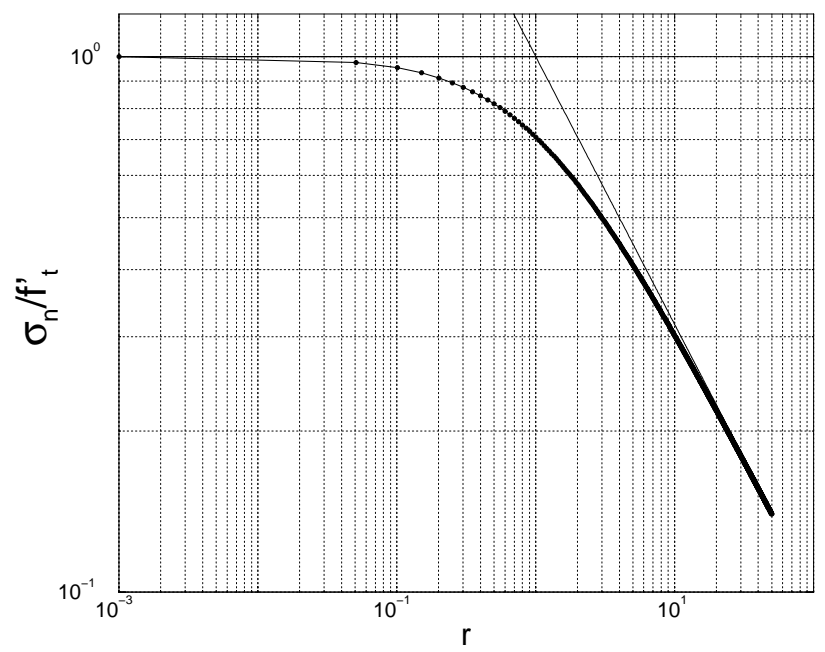

Figure 5. Size effect law for an edge crack with Dugdale's model.

Expressed in terms of $r=a_{0} / c_{f}, s=1 /(1+r)$, the far field nominal stress will be

$$
\sigma_{N}=(0.903 / \underbrace{\alpha}_{B}) f_{t}^{\prime}[1 /(1+\underbrace{r}_{\beta})]^{1 / 2} .
$$

For an infinite plate with an edge crack, $\alpha=1.1215$, and the resulting nominal stress will be

$$
\sigma_{N}=(\underbrace{0.805}_{B} f_{t}^{\prime})[1 /(1+\underbrace{r}_{\beta})]^{1 / 2} .
$$

This equation is shown in figure 5.

\section{Conclusion}

The size effect law is rederived through a balancing of far-field induced stress intensity factors with those caused by the cohesive stresses. Hence, a direct relationship between plasticity and LEFM to the size effect law is presented. It is shown that the shape of the cohesive stresses is irrelevant, that the $B$ coefficient can, albeit with some restriction, be analytically derived.

\section{References}

Barenblatt G 1962 The mathematical theory of equilibrium crack in the brittle fracture. Adv. Appl. Mech. 7: 55-125

Bažant Z P 1984 Size effect in blunt fracture: Concrete, rock, metal. J. Eng. Mech., Am. Soc. Civil Eng. 110: 518-535

Bažant Z, Planas J 1998 Fracture and size effect in concrete and other quasibrittle materials (Boca Raton, FL: CRC Press)

Broek D 1986 Elementary engineering fracture mechanics 4th edn (Dordrecht: Martinus Nijhoff) 
Cherepanov G 1979 Mechanics of brittle fracture (New York: McGraw-Hill)

Dugdale D 1960 Yielding of steel sheets containing slits J. Mech. Phys. Sol. 8: 100-108

Hillerborg A, Modéer M, Petersson P E 1976 Analysis of crack formation and crack growth in concrete by means of fracture mechanics and finite elements. Cement Concrete Res. 6: 773-782

Saouma V, Natekar D, Sbaizero O 2002 Nonlinear finite element analysis and size effect study of a metal-reinforced ceramics-composite. Mater. Sci. Eng. A323: 129-137

Stevens R, Guiu F 1994 The application of the $j$ integral to problems of crack bridging. Acta Metall. Mater 42: 1805-1810 